\title{
An Optic Determination Method of 2-Chlorophenol Based on Oxidation by Iron (III) Tetrasulfophthalocyanine Catalyze
}

\author{
Yilin Tong a, ${ }^{*}$, Xuecai Han, Peng Wu and Qiong Li \\ Hankou University, Wuhan, China \\ *, a529688591@qq.com
}

\begin{abstract}
An optic determination method based on the oxidation of 2-chlorophenol (2-CP) catalyzed by Iron (III) Tetrasulfophthalocyanine (Fe (III) PcTs) was developed for the determination of 2-CP. The optical oxygen sensing film containing fluorescence indicator $\mathrm{Ru}(\mathrm{bpy})_{3} \mathrm{Cl}_{2}$ was used to detect the consumption of oxygen in solution. A lock-in amplifier was used for detecting the lifetime of the oxygen sensing film by measuring the phase delay of the sensor head. Under the optimal conditions ( $\mathrm{pH} 6.0,25^{\circ} \mathrm{C}, \mathrm{Fe}$ (III) PcTs concentration of $0.62 \mathrm{mg} / \mathrm{mL}$ ), the linear detection range and response time of the biosensor were $1.0 \times 10^{-6}-9.0 \times 10^{-6} \mathrm{~mol} / \mathrm{L}$ and $230 \mathrm{~s}$, respectively. The sensor displayed high selectivity and could be used as an effective tool in analyzing 2-CP concentration in practical water samples.
\end{abstract}

Keywords: 2-chlorophenol, $\mathrm{Ru}(\mathrm{bpy})_{3} \mathrm{Cl}_{2}, \mathrm{Fe}$ (III) PcTs, oxygen, Iron (III).

\section{Introduction}

Chlorinated phenols are pervasive environmental pollutants because they are widely used in the production of several herbicides, pesticides, preservatives, plant growth regulators and formed in the environment as a result of the degradation and metabolism of these agricultural and food chemicals [1-3]. 2-chlorophenol (2-CP) is of perticular interest bacause it is a precursor for the synthesis of carcinogenic endocrine, 2-chlorophenolxyacetic acid, which is the active ingredient of more than 1500 herbicides. It had been regarded as the toxic organic substance to be specially controlled [4]. Therefore, the detection of 2-CP concerntion is very important to environmental protection and human health. Methords for the determination of 2-CP have included the gas chromatography [5, 6], flow injection analysis [7], HPLC [8], photocatalysis [9], electrode [10, 11], electrochemical sensor $[12,13]$, but these methords suffer from the disadvantages such as time-comsuming, high cost, complicated sample preparation and impossibility of on-line and real time detection. Fiber optic sensors have many advantages including fast response, high strong ability to resist disturbance, possibility of on-line and real time detection [14]. Fiber optic sensors based on enzyme catalysis provide the effective way for 2-CP detection. However, the natural enzymes have the drawbacks such as poor stability, very limited source, and difficult extraction and purification, which limit their applications.Iron(III) tetrasulfophthalocy-anine (Fe(III)PcTs) are the biomimetic enzyme which are stable and cost effective, and could be an effective replacement for the nature enzymes.

In our previous work, we studied the oxidation of 2, 4-DCP catalyzed by iron (III) tetrasulfophthalocyanine (Fe (III) PcTs). The influence of organic solvents, iron (III) tetrasulfophthalocyanine (Fe (III) PcTs) dosage, solution $\mathrm{pH}$ and temperature to the 2, 4-DCP oxidation were studied. A fiber optic sensor based on the 2, 4-DCP oxidation by oxygen with the catalysis of Fe (III) PcTs as a biomimetic enzyme has been designed, and the performance of the sensor was investigated. In this work, we fabricated a optic fiber sensor for determination of 2-chlorophenol based on oxygen oxidation catalyzed by Iron (III) Tetrasulfophthalocyanine, the sensor has the detection ranges of $1.0 \times 10^{-6} \sim 9.0 \times 10^{-6} \mathrm{~mol} / \mathrm{L}$ and the response time of $230 \mathrm{~s}$. The detection results of real samples by using this sensor indicate a promising application prospect. 


\section{Experimental}

\subsection{Reagents and Apparatus}

Fe (III) PcTs was synthesized and purified according to the literature [15].Fluorescence indicator ( $\mathrm{Ru}(\mathrm{bpy}){ }_{3} \mathrm{Cl}_{2}$ ) was purchased from Sigma-Aldrich. Cellulose acetate (CA) and DCP were purchased from Sinopharm Chemical Reagent Co., Ltd. Deionized water was used in all the experiments. All the reagents were of analytical grade and used without further purification.

A lock-in amplifier (SR830, Standford Research Systems, U. S. A) was used for measuring the phase delay of the sensor head.The absorption spectra were determined using a UV-2450 spectrophotometer (Shimadzu, Japan).

\subsection{Preparation of Optical Oxygen Sensing Film}

The oxygen sensing membrane was prepared by using $\mathrm{Ru}(\mathrm{bpy}){ }_{3} \mathrm{Cl}_{2}$ as the fluorescence indicator and CA as the matrix according to our previously work [16]. Briefly, $0.1 \mathrm{~g}$ cellulose acetate was added into $3.0 \mathrm{ml}$ acetone and stirred at room temperature for $2 \mathrm{~h}$. A certain amount of $\mathrm{Ru}(\mathrm{bpy})_{3} \mathrm{Cl}_{2}$ water solution was added in the mixture and it was stirred at room temperature for 6 hours to form an uniform solution. The solution was dipped onto a glass cluture dish with the diameter of $6 \mathrm{~cm}$ and makes it well-distributed. The cellulose acetate membrane embedded with fluorescent indicator was obtained after the acetone and water were volatilized.

\subsection{Preparation and Principle of Fiber Optic Sensor}

The detecting system consists of a lock-in amplifier, a LED with the excitation wavelength of 416 $\mathrm{nm}$ as the light source, a sensor head with a oxygen sensing membrane and a computer for data processing (see Fig. 1).

The principle of the sensor is based on the fluorescence quenching and consumption of oxygen [17]. The change of oxygen concentration was detected by measuring fluorescence of $\mathrm{Ru}(\mathrm{bpy}){ }_{3} \mathrm{Cl}_{2}$ quenched by oxygen. Since a lock-in amplifier is used, the quchening could be described as

$$
\frac{\tan \phi_{0}}{\tan \phi}=1+K_{s v}[Q]
$$

Where $\varphi_{0}$ and $\varphi$ are the phase delay change of the sensor in the absence and presence of the oxygen quencher, respectively, and Ksv is the Stern-Volmer constant. [Q] is oxygen concentration. Since $\varphi$ is very small (i.e. $<5^{\circ}$ ) in the experiment, $\tan \varphi \approx \varphi$. By collecting the data of phase delay $\varphi$ the quantification of 2-CP is achieved.

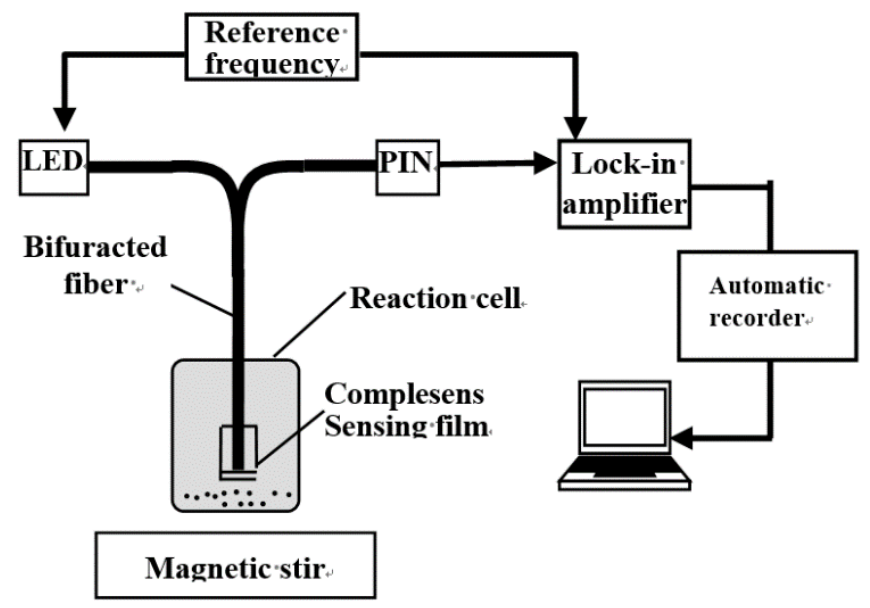

Figure 1. Schematic diagram of the detecting system 


\subsection{Measurements}

For detecting the 2-CP concentration, measurements were performed with the setup shown schematically in Fig. 1. The sensor head was placed into a tiny reaction cell which contained 4AAP aqueous solution $\left(1.0 \times 10^{-3} \mathrm{~mol} / \mathrm{L}\right), 2-\mathrm{CP}$ buffer solution $\left(1.0 \times 10^{-3} \mathrm{~mol} / \mathrm{L}\right.$ stock solution $)$ and a certain amount of Fe (III) PcTs powder $(31.0 \mathrm{mg})$. An entire airtight reaction cell was introduced to eliminate the interference of oxygen from the open air. All the measurements were carried out at $25^{\circ} \mathrm{C}$ with continuous and constant stirring. The fluorescence signal was collected by PIN and guided to the lock-in amplifier through the output bundle, and then transferred to phase-delay which was collected by the computer. The following measurement could be performed after a simple washing of the sensor head and Fe (III) PcTs with buffer solution. All the measurements were performed in tripicate.

\section{Results and Discussion}

\subsection{Dynamic Range of the Sensor}

This sensor was based on the fluorescence quenching and consumption of oxygen. The 2-CP concentration detection could be achieved by detecting the change of phase delay of the sensor head. In our previous work, we obtained the optimal conditions for the oxidation of 2-CP catalyzed by $\mathrm{Fe}(\mathrm{III}) P c T s$ : the solution $\mathrm{pH}$ was 6.0 , the Fe(III)PcTs amount was $0.62 \mathrm{mg} / \mathrm{mL}$, reaction temperature was $25^{\circ} \mathrm{C}$, ethanol amount was $5 \mathrm{~mL}$ (the total volume of system was $50 \mathrm{~mL}$ ). In this study we used these optimal conditions for the fiber optic 2-CP sensor based on Fe (III) PcTs catalysis. Ethanol was not added in the system because it could cause serious negetive effect to the optical oxygen sensing film.

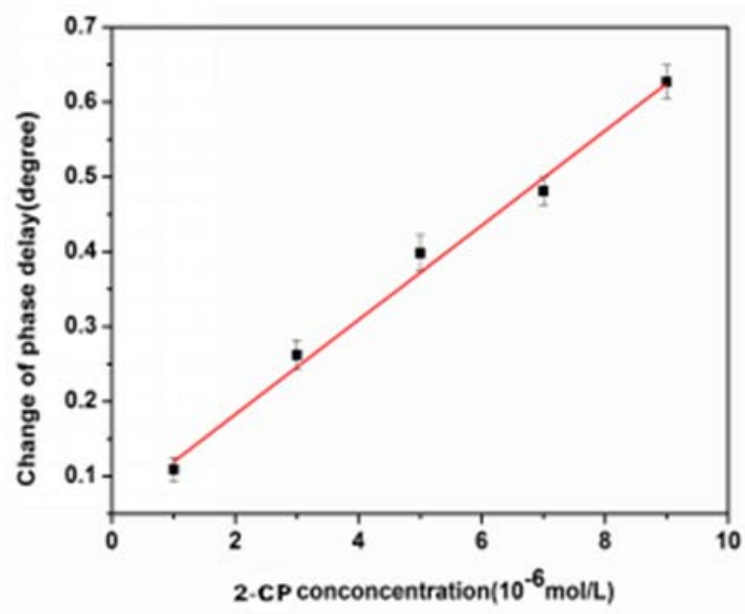

Figure 2. Typical calibration curve of the fiber optic 2-CP sensor at various concentration of 2CP.Conditions: $\mathrm{T}=25 \mathrm{oC}$, [Fe (III) PcTs] $=0.62 \mathrm{mg} / \mathrm{mL}, \mathrm{pH}=6.0$, Error bars in the figure show standard deviations calculated with three data points taken from different measurements.

Fig 2 shows the relationship between the change of phase delay $\varphi$ and 2-CP concentration in the range of $1.0 \times 10^{-6} \mathrm{~mol} / \sim 9.0 \times 10^{-6} \mathrm{~mol} / \mathrm{L}$ (The Integrated Wastewater Discharge Standard of China for DCP is $3.42 \times 10^{-6} \mathrm{~mol} / \mathrm{L}$ ). There is a good linear relationship between $\varphi$ and $2-\mathrm{CP}$ concentration. The linear graph was defined by the equation of $y=0.0542 x+0.0643$ and $R^{2}=0.981 . \varphi$ is defined as the difference between the phase delay of the sensor head with certain 2-CP concentration and with no $2-\mathrm{CP}$ in the solution. The response time of the sensor was taken as $230 \mathrm{~s}$ because most of the2-CP was oxidized in this time. The detection limit was $2.0 \times 10^{-7} \mathrm{~mol} / \mathrm{L}(\mathrm{S} / \mathrm{N}=3)$. For a solution with a certain 2-CP concentration, it is understandable that the detection is not influenced by the fluctuations of initial dissolved oxygen concentration in samples because the change of oxygen concentration is constant during the detection. Similar results were also reported previously. 


\subsection{Practical Application}

The fiber optic 2-CP sensor was validated by applying it to the determination of 2-CP in samples from water of East Lake and Yangtse River in Wuhan, China. The DCP concentrations in two samples were determined, in triplicate, using the proposed sensor. The results were shown in Table 1. It can thus be concluded that this sensor is suitable for the determination of 2-CP in practical samples.

Table 1. Detection of practical samples using fiber optic 2-CP sensor based on Fe (III) PcTs catalysis

\begin{tabular}{|c|c|c|c|}
\hline Samples & 2-CP added $\left(10^{-6} \mathrm{~mol} / \mathrm{L}\right)$ & 2-CP found $\left(10^{-6} \mathrm{~mol} / \mathrm{L}\right)(\mathrm{n}=3)$ & Recovery $(100 \%)$ \\
\hline Water in & 5.00 & $4.89 \pm 0.14$ & 97.2 \\
\hline River & & & \\
\hline Water in & 5.00 & $4.73 \pm 0.14$ & 96.2 \\
\hline East Lake & & & \\
\hline
\end{tabular}

\section{Conclusion}

The proposed sensor based on the oxidation by oxygen with Fe(III)PcTs catalysis is a precise, low cost, sensitive and highly selective device for the determination of 2-CP. The sensor was easily prepared, readily regenerated and demonstrated a long lifetime. It is first time to report that this sensor can be used for 2-CP determination. The sensor showed short response time, appropriate linear calibration curve, good selectivity and stability. Finally, the sensor can be successfully applied to detect 2-CP in real water sample, demonstrating a great application prospect.

\section{Acknowledgments}

This work was financially supported by Universities Excellent Young and Middle-aged Scientific and Innovation Team Project of Hubei Province (No T201630) and Hubei Provincal Department of Education Scientific Research Guidance Project (No B2016328).

\section{References}

[1]. R Mckinley, J A Plant, J N B Bell.Endocrine disrupting pesticides: Implications for risk assessment [J].Environment International, 2008, 34: 168-183.

[2]. J Wang, G Chen, M P Chatrathi.Capillary Electrophoresis Microchip with a Carbon NanotubeModified Electrochemical Detector [J]. Anal. Chem., 2004, 76: 298-302.

[3]. R. H Nicolette, T. W Tesfaye,A Omotayo.Microsomal cytochrome P450-3A4 (CYP3A4) nanobiosensor for the determination of 2, 4 - dichlorophenol-An endocrine disruptor compound [J].Electrochimica Acta, 2009, 54: 1925-1931.

[4]. Mehmood, Z.; Kelly, D. E.; Kelley, S. L. Cytochrome P450 3A4 mediated metabolism of 2, 4dichlorophenol [J]. Chemosphere. 1997, 34, 2281.

[5]. K Kasuga, M Tsutsui.Some new developments in the chemistry of metallophthalocyanines [J]. Coordination Chemistry Review, 1980; 32: 67-95.

[6]. Santana, C. M.; Padrón, M. E. T.; Ferrera, Z. S.; Rodríguez, J. J. S. J. Development of a solidphase microextraction method with micellar desorption for the determination of chlorophenols in water samples Comparison with conventional solid-phase microextraction method [J].Chromatogr. A. 2007, 1140, 13.

[7]. Huang, Y. P.; Li, J.; Ma, W. H.; Cheng, M. M.; Zhao, J. C.; Yu, J. C. J. Efficient H2O2 Oxidation of Organic Pollutants Catalyzed by Supported Iron Sulfophenylporphyrin under Visible Light Irradiation [J]. Phys. Chem. B. 2004, 108, 7263. 
[8]. O Dilsat, K Kagan, M Burcu.Heterostructured Fluorohectorite Clay as an Electrochemical Sensor for the Detection of 2,4-Dichlorophenol and the Herbicide 2,4-D [J].Chem. Mater., 2002, 14: $1755-1761$.

[9]. Muna, G. W.; Quaiserová-Mocko,V. and Swain, G. M. Chlorinated Phenol Analysis Using OffLine Solid-Phase Extraction and Capillary Electrophoresis Coupled with Amperometric Detection and a Boron-Doped Diamond Microelectrode [J]. Anal. Chem. 2005, 77, 6542.

[10]. $\quad \mathrm{R}$ A Doong, H C Tsai.Immobilization and characterization of sol-gel-encapsulated acetylcholinesterase fiber-optic biosensor[J]. Anal .Chim. Acta, 2001, 434: 239-246.

[11]. Ali, H.; Langlois, R.; Wagner, J. R.; Brasseur, N.; Paquette, B. Biological activities of phthalocyanines- $\mathrm{X}$ syntheses and analyses of sulfonated phthalocyanines [J]. Photochem. Photobiol. 1988, 47, 713.

[12]. Huang, J.; Fang, H.; Liu, C.; Gu, E. D. and Jiang, D. S. A Novel Fiber Optic Biosensor for the Determination of Adrenaline Based on Immobilized Laccase Catalysis [J]. Anal. Lett. 2008, 41,1430 .

[13]. Sorokin, A.; Seris, J. L.; Meunler, B. Study on Fiber Optic Gas Sensor Based on Fluorescence Quenching and Its Signal Detecting Method [J]. Science. 1995, 268, 1163.

[14]. Sorokin, A.; S. Suzzoni-Dezard, D.; Poullain, D.; Noel, J. P.; Meunier, Application of Lockin Amplifier in Optical Methane Gas Sensor. B [J]. J. Am. Chem. Soc. 1996, 118, 7410.

[15]. Hassanein, M.; Abdo, M.; Gerges, S.; El-khalafy, Modified dual lifetime referencing method for simultaneous optical determination and sensing of two analytes [J]. S. J. Mol. Catal. A: Chem. 2008, 287, 53.

[16]. W. M Grace, Q M Veronika, and M. S Greg.Chlorinated Phenol Analysis Using Off-Line Solid-Phase Extraction and Capillary Electrophoresis Coupled with Amperometric Detection and a Boron-Doped Diamond Microelectrode [J].Anal. Chem., 2005, 77: 6542-6548.

[17]. Li, D. P.; Tong, Y. L.; Huang, J.; Ding, L. Y.; Zhong, Y. M.; Zeng,D.; Yan, P. CO2 as the ultimate degradation product in the $\mathrm{H} 2 \mathrm{O} 2$ oxidation of 2,4,6-trichorophenol catalyzed by iron tetrasulfophthalocyanine [J] J. Mol. Cata. A: Chem. 2011, 345, 108. 